\title{
IMPORTÂNCIA DE OPORTUNIDADES DE DESENVOLVIMENTO E APOIO INSTITUCIONAL NA SATISFAÇÃO ACADÊMICA DE ENFERMEIROS
}

\author{
IMPORTANCE OF DEVELOPMENT OPPORTUNITIES \\ AND INSTITUTIONAL SUPPORT IN NURSES' ACADEMIC \\ SATISFACTION
}

\section{IMPORTANCIA DE OPORTUNIDADES DE DESARROLLO Y APOYO INSTITUCIONAL EN LA SATISFACCIÓN ACADÉMICA DE ENFERMEROS}

\author{
Francine da Costa Alves ${ }^{1}$ \\ Maíra Rosa Apostólico ${ }^{2}$ \\ Alfredo Almeida Pina-Oliveira ${ }^{3}$ \\ Ana Claudia Giesbrecht Puggina ${ }^{4}$
}

\begin{abstract}
Como citar este artigo: Alves FC, Apostólico MR, Pina-Oliveira AA, Puggina ACG. Importância de oportunidades de desenvolvimento e apoio institucional na satisfação acadêmica de enfermeiros. Rev baiana enferm. 2018;32:e25470.

Objetivos: identificar a satisfação acadêmica de enfermeiros assistenciais e associar características sociodemográficas, educacionais e profissionais do enfermeiro com a sua satisfação acadêmica. Método: estudo analítico transversal realizado com enfermeiros. Foram utilizados dois questionários: escala de satisfação com a experiência acadêmica de estudantes do ensino superior; e um questionário de caracterização. Resultados: a amostra do estudo foi constituída de 397 enfermeiros. Encontrou-se associações estatisticamente significativas nas dimensões Satisfação com o Curso e Oportunidade de Desenvolvimento com as variáveis sexo $(p<0,00)(p=0,04)$, escolaridade $(p=0,04)(p=0,02)$, tipo de instituição de ensino $(p=0,01)(p=0,04)$ e oportunidade em pesquisa $(p=0,02)(p<0,00)$. Conclusão: a satisfação acadêmica foi moderada. Enfermeiros do sexo masculino, que fizeram mestrado, estudaram em instituição pública e tiveram oportunidade em pesquisa, apresentaram-se satisfeitos com a graduação, organização do curso, atividades extracurriculares, relacionamentos com colegas, disponibilidade e conhecimento dos professores, e com a diversidade das atividades extracurriculares e currículo do curso oferecido.
\end{abstract}

Descritores: Enfermagem. Satisfação pessoal. Educação em enfermagem.

Objectives: identify the academic satisfaction of clinical nurses and associate the nurses' sociodemographic, educational and professional characteristics with their academic satisfaction. Method: cross-sectional analytic study

\footnotetext{
Enfermeira. Mestre em Enfermagem. Especialista em Docência em Enfermagem, Enfermagem do Trabalho e Acupuntura. Membro do Grupo Estudo e Pesquisa sobre Comunicação em Saúde da Universidade Guarulhos. Docente do Senac São Paulo. São Paulo, SP, Brasil. enffran@bol.com.br

2 Enfermeira. Doutora em Ciências. Pós-doutorado em Saúde Coletiva. Professora e Vice-Coordenadora do Programa de Pós-graduação em Enfermagem, Universidade Univeritas. Guarulhos, São Paulo, Brasil.

Enfermeiro. Doutor em Ciências. Especialista em Práticas de Promoção da Saúde. Educador em Saúde Pública no Centro de Promoção da Saúde do Hospital das Clínicas da Faculdade de Medicina da Universidade de São Paulo. Professor da Faculdade de Campo Limpo Paulista e do Programa de Pós-graduação em Enfermagem, Universidade Univeritas, Guarulhos, São Paulo, Brasil.

4 Enfermeira. Doutora em Ciências. Pós-doutorado em Comunicação Interpessoal. Fellowship no Coma Science Group da Universidade de Liege, Bélgica. Líder do grupo de pesquisa Estudo e Pesquisa sobre Comunicação em Saúde. Professora Adjunta da Faculdade de Medicina de Jundiaí, Professora e Coordenadora do Programa de Pós-graduação em Enfermagem da Universidade Univeritas, Guarulhos, São Paulo, Brasil.
} 
involving nurses. Two questionnaires were used: Scale of Higher Education Students' Satisfaction with Academic Experience; and a characterization questionnaire. Results: the study sample consisted of 397 nurses. Statistically significant associations between the dimensions Satisfaction with the Course and Development Opportunity and the variables $\operatorname{sex}(p<0.00)(p=0.04)$, education $(p=0.04)(p=0.02)$, type of teaching institution $(p=0.01)(p=0.04)$ and research opportunity $(p=0.02)(p<0.00)$. Conclusion: the academic satisfaction was moderate. Male nurses who took a Master's program, studied in a public institution and had research opportunities were satisfied with the undergraduate program, course organization, extracurricular activities, relationships with colleagues, teachers' availability and knowledge and with the range of extracurricular activities and course curriculum offered.

\section{Descriptors: Nursing. Personal satisfaction. Education, Nursing.}

Objetivos: identificarla satisfacción académica de enfermeros asistencialesy asociarcaracterísticas sociodemográficas, educativas y profesionales del enfermero con su satisfacción académica. Método: estudio analítico transversal, con enfermeros. Se utilizaron dos cuestionarios: escala de satisfacción con la experiencia académica de estudiantes de enseñanza superior; y cuestionario de caracterización. Resultados: muestra del estudio constituida de 397 enfermeros. Hubo asociación estadísticamente significativa en las dimensiones Satisfacción con el Curso y Oportunidad de Desarrollo con las variables sexo $(p<0,00)(p=0,04)$, escolaridad $(p=0,04)(p=0,02)(p=0,02)$, tipo de institución de enseñanza $(p=0,01)(p=0,04)$ y oportunidad en investigación $(p=0,02)(p<0,00)$. Conclusión: la satisfacción académica fue moderada. Los enfermeros del sexo masculino, que realizaron maestría, estudiaron en institución pública y tuvieron oportunidad en investigación, se presentaron satisfechos con la graduación, organización del curso, actividades extracurriculares, relaciones con colegas, disponibilidad y conocimiento de profesores, y con la diversidad de las actividades extracurriculares y curriculo del curso ofrecido.

Descriptores: Enfermería. Satisfacción personal. Educación en enfermería.

\section{Introdução}

As Instituições de Ensino Superior (IES), com o objetivo de gerar sucesso e satisfação acadêmica, devem disponibilizar recursos e proporcionar oportunidades para o desenvolvimento profissional ao longo do processo de formação inicial na graduação. Entretanto, sabe-se que o insucesso e as queixas de insatisfação dos alunos referem-se ao contexto e à organização do currículo acadêmico, bem como às expectativas idealizadas do curso ${ }^{(1)}$.

Os Projetos Pedagógicos de Curso (PPC) de Enfermagem definem as habilidades essenciais para a formação generalista do enfermeiro e devem traduzir aspectos epistemológicos e pedagógicos das Diretrizes Curriculares Nacionais (DCN). Estas recomendam a inserção precoce dos estudantes nos sistemas e serviços locais de saúde, a fim de motivá-los para a construção de uma identidade profissional, com base no e para o trabalho no âmbito do Sistema Único de Saúde (SUS). Para tanto, devem englobar estratégias de formação como estágios, metodologias ativas de aprendizagem, entre outras atividades complementares e de aperfeiçoamento do cuidado prestado à população ${ }^{(2)}$.

A satisfação com a experiência acadêmica é alcançada quando se atinge um resultado esperado, relacionado à expectativa do indivíduo (3) Por outro lado, observa-se menor desempenho do estudante, maior insegurança e aumento da possibilidade de abandono do curso quando a estrutura das IES não contempla as expectativas dos estudantes ${ }^{(4)}$. Sendo assim, conhecer as satisfações ou insatisfações dos estudantes pode auxiliar na compreensão sobre como a experiência acadêmica pode impactar na atuação do futuro profissional ${ }^{(4)}$.

A formação do enfermeiro deve contemplar o desenvolvimento de habilidades técnico-científicas, ético-políticas e relacionais com o intuito de sensibilizá-lo e prepará-lo para enfrentar as situações e constantes transformações no mundo do trabalho. Esta perspectiva está amparada pelas DCN, que enfatizam a importância de o futuro enfermeiro desempenhar suas atividades laborais com competências essenciais ${ }^{(5)}$. 
Enfermeiros satisfeitos com as vivências acadêmicas têm maior probabilidade de se considerarem bem-sucedidos e de sentirem satisfação profissional. Verifica-se que os fatores que têm maior relação com a satisfação acadêmica são aqueles que fornecem estímulos e oportunidades para que os alunos vivenciem na prática o que foi ensinado em sala de aula durante o curso de graduação em Enfermagem ${ }^{(6)}$.

Futuros enfermeiros relatam a necessidade de uma nova organização acadêmica comprometida com a formação de pessoas críticas, reflexivas e cidadãs com base em conhecimentos humanísticos e habilidades interpessoais que agregam valores sociais. Destarte, adquirir e reproduzir conhecimento torna-se insuficiente e os próprios estudantes de graduação requerem maior interação entre teoria e prática e maior aplicação de métodos ativos, dialógicos e participativos para a promoção de um aprendizado relevante ${ }^{(7)}$.

É importante compreender o significado da satisfação acadêmica e os respectivos fatores que influenciam positiva ou negativamente a trajetória acadêmica ${ }^{4}$. Na Enfermagem, este entendimento ajudará a atender as necessidades dos estudantes e alinhar suas expectativas em relação às oportunidades das IES para sua formação, além da correspondência com as DCN. Trata-se de um processo contínuo entre identificar aspectos que poderão ser melhorados e reformulados de acordo com o resultado da satisfação acadêmica e realinhar os PPC e a estrutura institucional.

Frente a isso, os objetivos deste estudo foram identificar a satisfação acadêmica de enfermeiros e associar características sociodemográficas, educacionais e profissionais do enfermeiro com a sua satisfação acadêmica.

\section{Método}

Estudo analítico transversal, que adotou a satisfação acadêmica como variável dependente e as características sociodemográficas, educacionais e profissionais como variáveis independentes.
Os participantes do estudo foram enfermeiros que atuavam ou já atuaram na área assistencial e tinham perfil ativo na rede social Facebook®. A coleta de dados foi realizada em ambiente virtual com enfermeiros pertencentes a três hospitais públicos do interior de São Paulo, e com enfermeiros que participavam de grupos públicos relacionados à enfermagem na rede social Facebook ${ }^{\circledR}$.

Para o recrutamento direto, os enfermeiros foram abordados no ambiente de trabalho, após autorização das instituições e do Comitê de Ética em Pesquisa. Foram esclarecidos sobre a pesquisa e convidados a participar. Após o aceite, informaram o nome de perfil na rede social Facebook ${ }^{\circledR}$, por onde receberam um convite individual via mensagem e um link para acesso ao questionário.

O recrutamento indireto ocorreu de duas maneiras. Foi publicado um link na linha do tempo de grupos públicos relacionados à Enfermagem, na rede social Facebook ${ }^{\circledR}$, convidando enfermeiros a participarem da pesquisa. Adicionalmente, enfermeiros com perfis pessoais vinculados aos pesquisadores foram convidados a colaborar com a pesquisa por mensagem privada.

Para garantir a veracidade das informações e prevenir duplicidade de respostas, solicitou-se a identificação com nome completo e número de inscrição no Conselho Regional de Enfermagem (COREN). Os participantes cujos registros não foram localizados nos sites dos conselhos regionais foram excluídos da amostra. Para os questionários respondidos mais de uma vez, foi mantido apenas o primeiro envio do participante.

A coleta de dados ocorreu entre junho e novembro de 2016 e todos os participantes responderam a dois questionários disponibilizados no Google Drive®. A primeira tela após acesso ao instrumento foi o Termo de Consentimento Livre e Esclarecido (TCLE). O consentimento foi obtido por meio de aceite eletrônico. Para tanto, o participante deveria clicar em "aceito participar da pesquisa" para prosseguir. Caso a escolha fosse "não aceito participar da pesquisa", 
o participante seria direcionado a uma página de agradecimento, finalizando sua participação.

O primeiro questionário, de caracterização dos participantes, continha variáveis sociodemográficas (idade, naturalidade, sexo, estado civil, cor de pele autorreferida, religião, renda pessoal), educacionais (escolaridade completa, instituição de nível superior que cursou Enfermagem, participação em atividades acadêmicas) e profissionais (tempo de formação, formação prévia na área de enfermagem como auxiliar ou técnico de enfermagem, turno de trabalho e unidade de atuação).

O segundo questionário utilizado foi a Escala de Satisfação com a Experiência Acadêmica de Estudantes do Ensino Superior (ESEA), composta por 35 itens divididos em três dimensões (satisfação com o curso, oportunidade de desenvolvimento e satisfação com a instituição). As respostas são do tipo Likert de cinco pontos, que variam de "nada satisfeito" a "totalmente satisfeito". O escore total da escala varia de 55 a 175 , sendo maior a satisfação com a experiência acadêmica quanto maior for o escore ${ }^{6}$.

Foi realizada análise descritiva (frequência absoluta, relativa, medidas de posição e desvio-padrão) e inferencial. Os dados foram analisados pelo programa estatístico $R$ Core Team 2016®.

Para a comparação das variáveis quantitativas com os escores do instrumento foi utilizado o Teste de Correlação de Spearman; na associação das variáveis categóricas com os escores foram utilizados o Teste de Kruskal-Wallis e o Teste de Mann-Whitney. O nível de significância adotado nos testes foi de 0,05. Quando o teste da associação foi significativo, foram analisados os p-valores nas comparações dois a dois pelo critério de Bonferroni; os grupos diferentes entre si são aqueles menores que o p-valor geral.

O desenvolvimento do estudo atendeu às normas nacionais e internacionais de ética em pesquisa envolvendo seres humanos e foi aprovado pelo Parecer n. 1.562.760 do Comitê de Ética e Pesquisa da Universidade Guarulhos (UNG).

\section{Resultados}

A amostra do estudo foi constituída de 397 enfermeiros com média de idade de 34,72 anos $( \pm 8,10)$, com tempo médio de formação de 6,84 $( \pm 5,15)$. A maioria era do sexo feminino $(n=363$; 91,44\%), católicos $(n=216 ; 54,41 \%)$ e brancos $(n=281 ; 70,78 \%)$; provenientes da região Sudeste $(n=337 ; 84,89 \%)$. As maiores frequências foram de pessoas casadas $(n=195 ; 49,12 \%)$, com renda de até 3 salários mínimos ( $n=116 ; 29,22 \%)$.

A maioria dos profissionais concluiu graduação em universidades privadas $(n=331$; 83,38\%), cursou especialização ( $n=212 ; 53,40 \%)$, não tinha formação prévia na área de enfermagem antes da graduação $(n=218 ; 54,91 \%)$. Menos da metade participou de eventos científicos durante a graduação $(n=136 ; 34,26 \%)$, atuava e/ou atuou em unidades de internação $(n=165 ; 41,56 \%)$ e em turno de trabalho integral de 8 h diárias $(n=148 ; 37,28 \%)$.

O escore médio total das respostas dos enfermeiros em relação a ESEA, foi de 113,57( $\pm 20,62)$, discretamente acima do ponto médio do instrumento (105), o que indica uma satisfação acadêmica moderada (Tabela 1). 
Tabela 1 - Descrição do escore total e das dimensões da Escala de Satisfação com a Experiência Acadêmica de Estudantes do Ensino Superior. Guarulhos, São Paulo, Brasil, 2016. (N=397)

\begin{tabular}{l|c|c|c|c|c|c|c}
\hline Variáveis & $\begin{array}{c}\text { Número } \\
\text { de itens }\end{array}$ & $\begin{array}{c}\text { Variação } \\
\text { do escore }\end{array}$ & Média & $\begin{array}{c}\text { Desvio- } \\
\text {-padrão }\end{array}$ & Mínimo & Mediana & Máximo \\
\hline $\begin{array}{l}\text { Satisfação com } \\
\text { o curso }\end{array}$ & 13 & $13-65$ & 44,92 & 8,02 & 28 & 43 & 65 \\
$\begin{array}{l}\text { Oportunidade de } \\
\text { desenvolvimento }\end{array}$ & 10 & $10-50$ & 30,60 & 6,42 & 16 & 30 & 50 \\
$\begin{array}{l}\text { Satisfação com } \\
\text { a instituição }\end{array}$ & 12 & $12-60$ & 38,04 & 8,07 & 19 & 36 & 60 \\
Total & $\mathbf{3 5}$ & $\mathbf{3 5 - 1 7 5}$ & $\mathbf{1 1 3 , 5}$ & $\mathbf{2 0 , 5}$ & $\mathbf{6 9}$ & $\mathbf{1 0 9}$ & $\mathbf{1 7 5}$ \\
\hline
\end{tabular}

Fonte: Elaboração própria.

Os itens da ESEA com as maiores médias estavam relacionados à dimensão "Satisfação com o curso", mais especificamente nas relações com colegas e professores, satisfação com o conhecimento dos docentes sobre o conteúdo das disciplinas que ministram e o envolvimento pessoal nas atividades do curso. Os itens da ESEA com as menores médias focaram na dimensão Oportunidade de desenvolvimento, apontado uma insatisfação dos alunos em relação ao papel institucional de gerar oportunidades de desenvolvimento pessoal, realizar eventos sociais e programas de apoio (Tabela 2 ).

Tabela 2 - Análise descritiva dos itens da Escala de Satisfação com a Experiência Acadêmica do Estudante do Ensino Superior. Guarulhos, São Paulo, Brasil, 2016. (N=397) (continua)

\begin{tabular}{|c|c|c|c|}
\hline \multicolumn{4}{|c|}{ Dimensão 1 - Satisfação com o curso } \\
\hline Itens & Afirmativas & Média & $\begin{array}{l}\text { Desvio } \\
\text { Padrão }\end{array}$ \\
\hline 5 & Relacionamento com os colegas do curso & 3,70 & 0,86 \\
\hline 13 & $\begin{array}{l}\text { Conhecimento dos professores sobre o conteúdo das disciplinas que } \\
\text { ministram }\end{array}$ & 3,70 & 0,80 \\
\hline 1 & Relacionamento com os professores & 3,61 & 0,82 \\
\hline 12 & Interesse dos professores em atender os estudantes durante as aulas & 3,51 & 0,84 \\
\hline 33 & Disponibilidade dos professores em atender os alunos fora da sala de aula & 3,51 & 0,84 \\
\hline 8 & $\begin{array}{l}\text { Adequação entre o envolvimento pessoal no curso e o desempenho } \\
\text { acadêmico obtido }\end{array}$ & 3,47 & 0,80 \\
\hline 31 & Relevância do conteúdo das disciplinas & 3,44 & 0,80 \\
\hline 35 & $\begin{array}{l}\text { Adequação entre as tarefas exigidas e o tempo estabelecido pelos } \\
\text { professores para realização }\end{array}$ & 3,44 & 0,80 \\
\hline 14 & $\begin{array}{l}\text { Reconhecimento por parte dos professores do meu envolvimento com } \\
\text { minha formação }\end{array}$ & 3,43 & 0,82 \\
\hline 21 & Compromisso da instituição com a qualidade da formação & 3,34 & 0,88 \\
\hline 34 & Adequação do conteúdo para formação & 3,34 & 0,88 \\
\hline 28 & Estratégia de aula utilizada pelos professores & 3,22 & 0,78 \\
\hline 25 & Avaliação proposta pelos professores & 3,16 & 0,73 \\
\hline
\end{tabular}


Tabela 2 - Análise descritiva dos itens da Escala de Satisfação com a Experiência Acadêmica do Estudante do Ensino Superior. Guarulhos, São Paulo, Brasil, 2016. (N=397) (conclusão) Dimensão 2 - Oportunidade de desenvolvimento

\begin{tabular}{l|l|cc}
\hline Itens & \multicolumn{1}{c|}{ Affirmativas } & Média & $\begin{array}{c}\text { Desvio } \\
\text { Padrão }\end{array}$ \\
\hline 9 & Envolvimento pessoal nas atividades do curso & 3,63 & 0,76 \\
3 & Currículo do curso & 3,41 & 0,78 \\
26 & Adequação entre o meu investimento financeiro para custear os estudos & 3,19 & 0,89 \\
& e a formação recebida & 3,09 & 0,95 \\
2 & Diversidade das atividades extracurriculares oferecidas pela instituição & 3,06 & 0,90 \\
11 & Condições oferecidas para o meu desenvolvimento profissional & 3,05 & 0,99 \\
17 & Condições para ingresso na área profissional de formação & 2,87 & 0,92 \\
24 & Oportunidade de desenvolvimento pessoal oferecida pela instituição & 2,84 & 0,92 \\
6 & Eventos sociais oferecidos pela instituição & 2,74 & 0,89 \\
10 & Programas ou serviços de apoio aos estudantes oferecidos pela instituição & 2,67 & 1,09 \\
23 & Programa de apoio financeiro oferecido pela instituição & & \multicolumn{1}{c}{. } \\
\hline
\end{tabular}

\begin{tabular}{|c|c|c|c|}
\hline \multicolumn{4}{|c|}{ Dimensão 3 - Satisfação com a instituição } \\
\hline Itens & Afirmativas & Média & $\begin{array}{l}\text { Desvio } \\
\text { Padrão }\end{array}$ \\
\hline 27 & Limpeza da instituição & 3,47 & 0,86 \\
\hline 18 & Acervo disponível na biblioteca & 3,35 & 0,95 \\
\hline 4 & Recursos e equipamentos audiovisuais disponíveis na instituição & 3,28 & 0,89 \\
\hline 16 & $\begin{array}{l}\text { Atendimento e clareza das informações oferecidas pelos funcionários da } \\
\text { biblioteca }\end{array}$ & 3,25 & 0,97 \\
\hline 29 & Serviços oferecidos pela biblioteca & 3,22 & 0,84 \\
\hline 22 & Infraestrutura física da instituição & 3,17 & 0,87 \\
\hline 32 & Localização dos diferentes setores que compõem a instituição & 3,17 & 0,87 \\
\hline 19 & Segurança oferecida pela instituição & 3,10 & 0,96 \\
\hline 20 & Infraestrutura física das salas de aula & 3,05 & 0,91 \\
\hline 30 & Conforto das instalações da instituição & 3,04 & 0,85 \\
\hline 7 & $\begin{array}{l}\text { Atendimento e clareza das informações oferecidas pelos funcionários da } \\
\text { secretaria }\end{array}$ & 3,00 & 0,93 \\
\hline 15 & Equipamentos e softwares oferecidos pelo laboratório de informática & 2,90 & 0,99 \\
\hline
\end{tabular}

Fonte: Elaboração própria.

Houve correlação estatisticamente significativa e negativa na comparação da dimensão Oportunidade de Desenvolvimento com o tempo de formação do enfermeiro. Quanto maior era o tempo de formado mais insatisfação foi apresentada em relação à responsabilidade institucional de oferecer atividades extracurriculares, currículo do curso mais adequado à prática e ao mercado de trabalho e apoio acadêmico (Tabela 3).

Tabela 3 - Correlação entre as dimensões da Escala de Satisfação com a Experiência Acadêmica do Estudante do Ensino Superior e o tempo de formação e idade. Guarulhos, São Paulo, Brasil, 2016. $(\mathrm{N}=397)$

\begin{tabular}{l|c|c|c|c}
\hline ESEA & Tempo de Formação & \multicolumn{2}{c}{ Idade } \\
\hline Dimensões & r & p-valor & r & p-valor \\
\hline Satisfação com o curso & $-0,07$ & 0,14 & -0.06 & 0,20 \\
Oportunidade de desenvolvimento & $-0,10$ & 0,04 & $-0,06$ & 0,18 \\
Satisfação com a instituição & $-0,07$ & 0,15 & 0,01 & 0,74 \\
\hline
\end{tabular}

Fonte: Elaboração própria. 
Houve associações estatisticamente significativas nas dimensões Satisfação com o curso e Oportunidade de desenvolvimento com sexo, escolaridade, tipo de instituição de ensino e oportunidade em pesquisa. Os achados mostraram que os enfermeiros do sexo masculino, que fizeram mestrado, estudaram em instituição pública e tiveram alguma oportunidade em pesquisa foram os mais satisfeitos com a graduação, organização do curso, atividades extracurriculares, relacionamento com professores colegas de curso, disponibilidade e conhecimento dos professores, além de mais satisfeitos também com a diversidade das atividades extracurriculares e currículo do curso oferecido pela instituição de ensino (Gráficos 1 e 2).

Gráfico 1 - Associações estatisticamente significativas das variáveis sexo e escolaridade com a Experiência Acadêmica de Estudantes do Ensino Superior. Guarulhos, São Paulo, Brasil, 2016. (N=397)
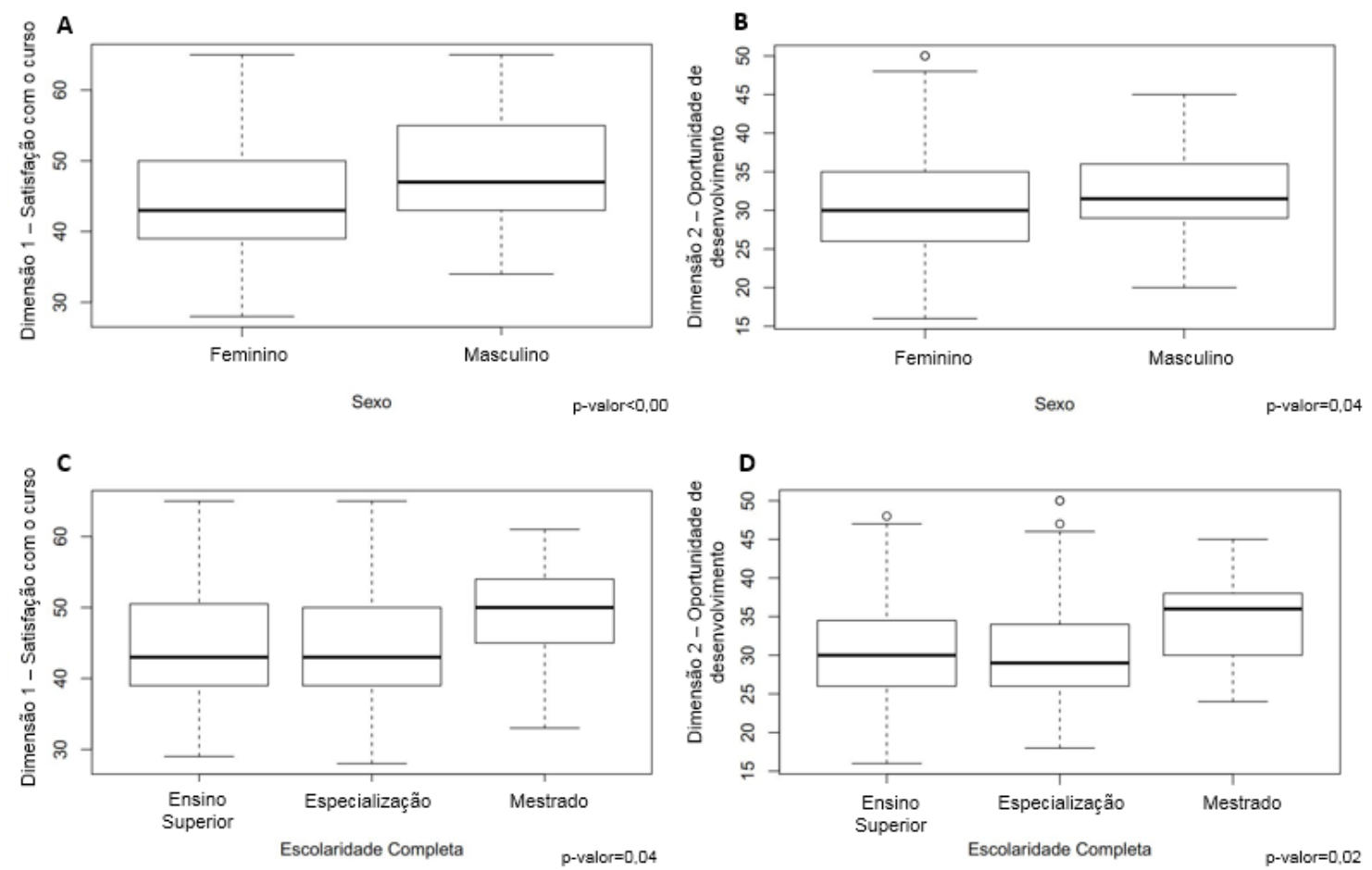

Fonte: Elaboração própria.

Legenda: p-valor: nível de significância. Resultado da comparação dois-a-dois pelo método Bonferroni: C: mestrado versus superior (p-valor 0,02); mestrado versus especialização (p-valor 0,01). D: mestrado versus superior (p-valor 0,01); mestrado versus especialização (p-valor 0,00). A e B: Teste Mann-Whitney. C e D: Teste Kruskal-Wallis. 
Gráfico 2 - Associações estatisticamente significativas das variáveis tipo de instituição de formação e oportunidades em pesquisa com a Experiência Acadêmica de Estudantes do Ensino Superior. Guarulhos, São Paulo, Brasil, 2016. (N=397)
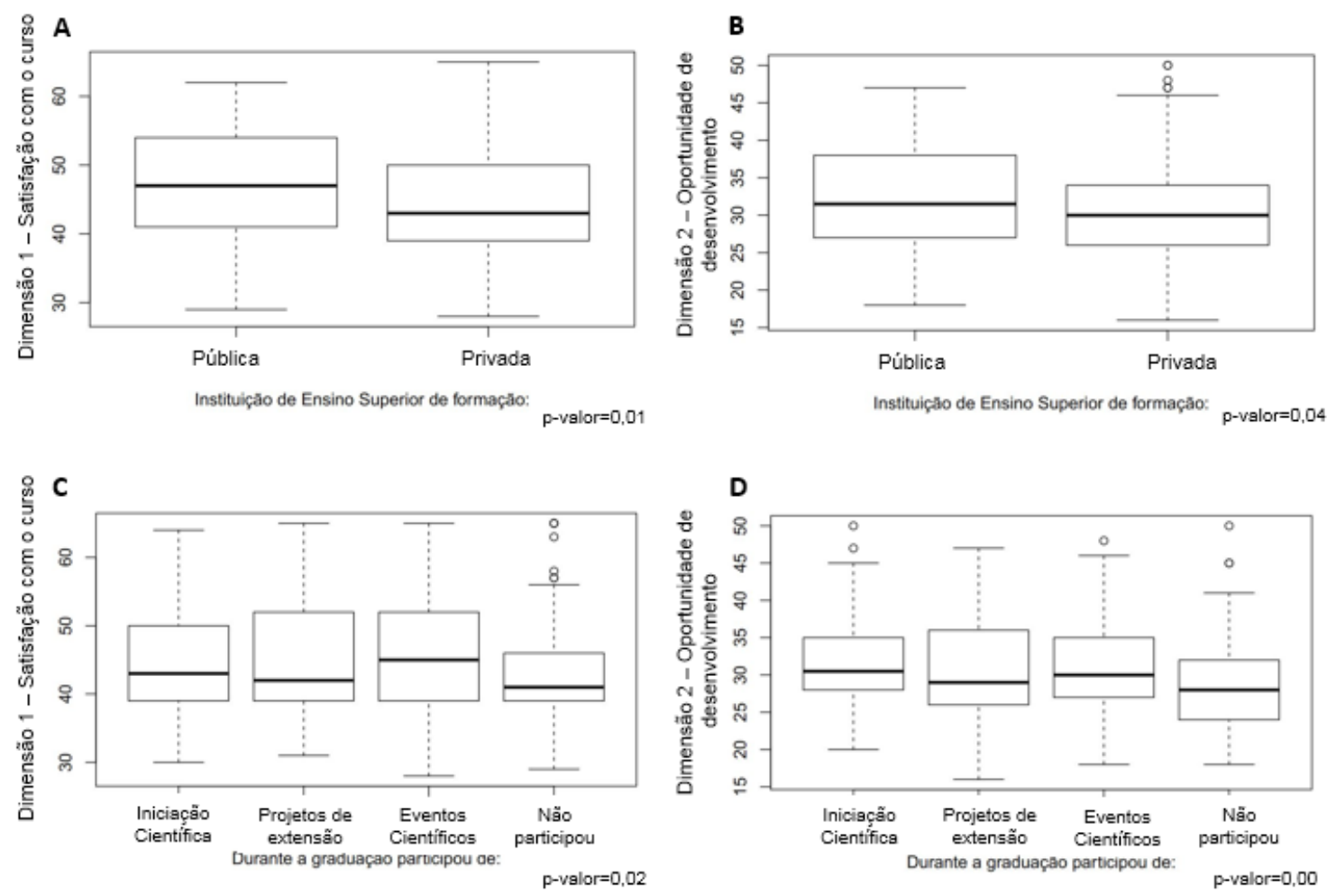

Fonte: Elaboração própria.

Legenda: p-valor: nível de significância. Resultado da comparação dois-a-dois pelo método Bonferroni: C: não participou de atividades de pesquisa versus demais (p-valor 0,00). D: não participou de atividades de pesquisa versus iniciação científica (p-valor 0,00). A e B: Teste Mann-Whitney. C e D: Teste Kruskal-Wallis.

\section{Discussão}

Os enfermeiros que participaram deste estudo mostraram satisfação acadêmica moderada, com maiores médias nos itens relativos às relações com colegas e professores, conhecimento dos docentes e envolvimento pessoal nas atividades do curso. Entretanto, houve insatisfação nos itens referentes à oportunidade de desenvolvimento relacionado ao papel institucional de gerar oportunidades de desenvolvimento pessoal, realizar eventos sociais e programas institucionais de apoio aos alunos.

Autores ${ }^{(8-10)}$ evidenciam a importância do professor na satisfação acadêmica. O aluno que ingressa na universidade tem perspectiva sobre o que irá aprender durante a graduação e quer ser estimulado por meio de trabalhos e integração entre as disciplinas para se interessar e sentir-se satisfeito $^{(8)}$.

O professor precisa compreender a magnitude e responsabilidade do seu papel. A busca permanente por conhecimentos, a clareza em relação a sua posição no mundo em constante transformação e o senso crítico para classificar e selecionar diferentes tecnologias (presenciais e/ou digitais) convergem no desenvolvimento de habilidades necessárias para o ensino, habilitando-se para exercer a docência com eficiência. Quanto às instituições, cabe-lhes proporcionar a melhoria contínua desses profissionais ${ }^{(9)}$.

A formação profissional do professor influencia sobremaneira na aprendizagem dos alunos, em especial quando o relacionamento que se cria entre eles é solidário, o ambiente favorável à aprendizagem e a diversidade de 
materiais educativos favorece a aprendizagem e gera um efeito positivo e cumulativo de satisfação e motivação dos estudantes de Enfermagem $^{(10)}$. Entretanto, ressalta-se, no estudo citado, que os enfermeiros com maior tempo de formação relataram maior insatisfação com a responsabilidade das IES no tocante à oferta de atividades extracurriculares, ao currículo do curso não adequado à prática profissional e à potencial inserção no mercado de trabalho. Esses resultados ${ }^{(10)}$ e os de outros estudos ${ }^{(11-13)}$ coincidem com os encontrados neste estudo, que avança na compreensão e na necessidade de fomentar oportunidades para os estudantes de graduação em Enfermagem nessas áreas.

A participação em programas de pesquisa, extensão e iniciação científica contribui para a formação profissional, uma vez que possibilita o envolvimento discente em atividades extracurriculares que enfatizam o aprendizado e enriquecimento do seu currículo, para atuar na prática profissional $^{(11)}$.

A busca dos alunos pelas atividades extracurriculares justifica-se por reconhecerem a necessidade de assumir maior responsabilidade pelo seu percurso profissional presente e futuro. Alguns percebem que nem todos os conteúdos necessários à sua formação podem ser trabalhados em sala de aula e buscam esse conhecimento por meio de alternativas como atividades extracurriculares no âmbito da extensão e da pesquisa ${ }^{(12)}$.

Oferecer atividades extraclasse auxilia na capacitação do aluno para desenvolver raciocínio crítico e na adequada percepção de si, da realidade, do mercado de trabalho e de sua projeção profissional. Nesse sentido, tais atividades contribuem para o autoconhecimento necessário ao aluno para construir seu perfil profissional, aumentar sua capacidade analítica e desenvolver sua identidade profissional ${ }^{(13)}$.

Outro resultado relevante deste estudo foi identificar que enfermeiros que fizeram mestrado, estudaram em instituições públicas e tiveram oportunidade em pesquisa são mais satisfeitos com a graduação e com as oportunidades de desenvolvimento oferecidas pelas instituições.
Autores reforçam o papel fundamental da universidade na formação do aluno, ao oferecerem oportunidades para o desenvolvimento deles tanto em sala de aula quanto em atividades extraclasse, e contribuírem para a sua futura inserção em um mundo do trabalho em constantes transformações e que exige do futuro profissional uma capacidade crítica, reflexiva e construtiva ${ }^{(13)}$.

Uma perspectiva positiva está em considerar o uso das metodologias ativas no processo educativo em Enfermagem. Ao auxiliarem na associação entre teoria e prática, essas metodologias favorecem o enfrentamento de situações desafiadoras no ambiente de trabalho, que exigem o raciocínio crítico, a criatividade e a tomada de decisões. Incorporar boas práticas de ensino, que resultem na aprendizagem significativa, extrapola o saber conhecer. Ao desenvolver as dimensões do saber agir, saber ser e saber conviver com os outros desde o início da formação acadêmica, os alunos são provocados a fazer reflexões sobre seu papel social e seu potencial profissional $^{(14)}$.

A aprendizagem baseada em problemas (ABP) exemplifica uma dessas metodologias ativas que contribuem para uma alta satisfação em estudantes de Enfermagem, mas demandam das IES mudanças organizacionais robustas. Contudo, após a implantação de aulas práticas com a ABP, pesquisadores identificaram 78\% de aprovação dos alunos, com relatos de alta satisfação com essa nova abordagem de ensino, recursos educacionais disponíveis e conteúdos apresentados de forma útil, prática e estimulante para o desenvolvimento e a responsabilidade discente ${ }^{(15)}$.

Em relação às práticas clínicas do curso de Enfermagem, estudo ${ }^{(16)}$ demonstrou que a maioria dos alunos (70\%) sente-se satisfeita com as atividades de ensino nos campos de prática e estágios, bem como com o comportamento dos professores em estimular, dar suporte durante as práticas e facilitar o aprendizado em serviços ${ }^{(16)}$.

Os achados deste estudo remetem a questões importantes que ocorreram no ensino superior brasileiro e que, direta ou indiretamente, relacionam-se com a satisfação acadêmica: o aumento 
10

Importância de oportunidades de desenvolvimento e apoio institucional na satisfação acadêmica de enfermeiros

no número de cursos de graduação em Enfermagem e a exigência de qualificação docente para os desafios da formação da força de trabalho em Enfermagem. Houve um avanço potencial nas últimas décadas com mudanças importantes tanto no funcionamento institucional, na configuração curricular, na titulação dos docentes, na institucionalização da pesquisa, na produção intelectual, na qualidade da formação oferecida, quanto na diversidade de ofertas de cursos, aumento no número de estudantes e, consequentemente, necessidade de aumento do corpo docente ${ }^{(17)}$.

Considerando o período de 1991 a 2012, a expansão dos cursos no território brasileiro foi de 393\%. A região Sudeste abrange $43 \%$ do total de cursos de graduação em Enfermagem no país, $36,73 \%$ desse percentual só em São Paulo, seguida da região Nordeste com 24,12\%, região Sul com 14,61\%, Centro-Oeste com 10,96\%, e Região Norte com 7,3\% ${ }^{(18-19)}$. No ano de 2016, os cursos de graduação em Enfermagem totalizavam 689.

A Enfermagem tornou-se uma das cinco profissões mais procuradas no Brasil e a situação desse crescimento desordenado exige uma análise crítica, pois os cursos tornaram-se caros e a qualidade da formação preocupante ${ }^{(18-19)}$, refletindo negativamente no processo de trabalho desse futuro profissional ${ }^{(19-20)}$. Apesar das DCN do Curso de Graduação em Enfermagem preconizarem a formação generalista, humanista, crítica e reflexiva para a construção do perfil do enfermeiro brasileiro, a exigência para o corpo docente é que seja constituído por Especialistas, Mestres e Doutores, não somente conhecedores de uma determinada área de atuação ${ }^{(21)}$. O aumento do número de instituições, por vezes sem controle ou garantia da qualidade, justifica a percepção de falta de apoio institucional. Por outro lado, a exigência de um número mínimo de Mestres e Doutores nos cursos de graduação pode ter refletido na satisfação acadêmica, no que se refere à relação com os docentes e com o domínio do conteúdo expresso nas aulas.

Esse aumento de IES favorece a constituição de vínculos trabalhistas mais frágeis para o corpo docente, limitando as inserções de estudantes de graduação em Enfermagem a uma formação mais integral, com oportunidades em pesquisa e extensão. A expansão do número de IES demanda um grande esforço na definição de padrões de funcionamento, investimento e qualidade. A missão do ensino superior deve resguardar o desenvolvimento de pesquisas científicas, por exemplo, por implicar sobremaneira na construção de uma identidade pedagógica, administrativa e institucional ${ }^{(22)}$.

Diante da relação desfavorável verificada entre o crescimento da oferta de cursos de Enfermagem e a satisfação acadêmica, torna-se essencial que os profissionais que atuam nas IES comprometam-se com a valorização da atuação dos profissionais da educação e a implementação das políticas para a melhoria do ensino superior $^{(23)}$.

Apesar de os resultados deste estudo mostrarem um contexto de profissionais formados majoritariamente em instituições de ensino privadas, o fato de se aproximar de maneira fiel da realidade e das condições do ensino superior no Brasil evidencia a sua importância.

\section{Conclusão}

Neste estudo, a satisfação acadêmica foi moderada, quando se avaliou, de maneira mais positiva, as relações desenvolvidas no ambiente universitário e o domínio de conhecimento dos docentes. Os motivos de insatisfação foram relacionados ao apoio institucional e à falta de oportunidades de desenvolvimento.

Os enfermeiros do sexo masculino, que fizeram mestrado, estudaram em instituição pública e tiveram oportunidade de se envolver em pesquisa, apresentaram-se satisfeitos com a graduação, organização do curso, atividades extracurriculares, relacionamentos com colegas, disponibilidade e conhecimento dos professores, e com a diversidade das atividades extracurriculares e currículo do curso oferecido.

A insatisfação foi maior entre os enfermeiros com maior tempo de formação e estava relacionada com as oportunidades oferecidas pela instituição, as atividades extracurriculares 
disponibilizadas, o currículo do curso pouco condizente com as atividades práticas e o mercado de trabalho, além do apoio acadêmico.

\section{Colaborações:}

1. concepção, projeto, análise e interpretação dos dados: Francine da Costa Alves e Ana Claudia Giesbrecht Puggina;

2. redação do artigo e revisão crítica relevante do conteúdo intelectual: Francine da Costa Alves, Maíra Rosa Apostólico, Alfredo Almeida Pina-Oliveira e Ana Claudia Giesbrecht Puggina;

3. aprovação final da versão a ser publicada: Francine da Costa Alves, Maíra Rosa Apostólico, Alfredo Almeida Pina-Oliveira e Ana Claudia Giesbrecht Puggina.

\section{Referências}

1. Porto MAS, Soares AB. Diferenças entre expectativas e adaptação acadêmica de universitários de diversas áreas do conhecimento. Anál Psicol [Internet]. 2017 [cited 2017 June 23];31(1):13-24. Available from: http://dx.doi.org/10.14417/ap.1170

2. Lopes Neto D, Teixeira E, Vale EG, Cunha FS, Xavier IM, Fernandes JD, et al. Aderência dos cursos de graduação em enfermagem as diretrizes curriculares nacionais. Rev bras enferm [Internet]. 2007 [cited 2017 June 23];60(6):627-34. Available from: http://dx.doi.org/10.1590/S0034-7 1672007000600003

3. Carvalho G, Lopes S. Satisfação profissional do enfermeiro em uma unidade de emergência de hospital geral. Arq Ciênc Saúde [Internet]. 2006 [cited 2015 Dec 20];13(4):215-19. Available from: http://repositorio-racs.famerp.br/racs_ol/vol-13-4/ Famerp\%2013(4)\%20ID\%20210\%20-\%2017.pdf

4. Schleich ALG, Polydoro SAJ, Santos AAA. Escala de satisfação com a experiência acadêmica de estudantes do ensino superior. Aval Psicol [Internet]. 2006 [cited 2015 Dec 6];5(1):11-20. Available from: http://pepsic.bvsalud.org/pdf/avp/ v5n1/v5n1a03.pdf

5. Vieira MA, Souto LES, Souza SM, Lima CA, Ohara CVS, Domenico EBL. Diretrizes curriculares nacionais para a área da enfermagem: o papel das competências na formação do enfermeiro. Renome [Internet]. 2016 [cited 2017 June 23];5(1);105-21.
Available from: http://www.renome.unimontes.br/ index.php/renome/article/view/102/148

6. Branquinho NCSS. Satisfação dos egressos do curso de graduação em enfermagem de uma universidade pública [dissertação]. Goiânia: Faculdade de Enfermagem, Universidade Federal de Goiás; 2012.

7. Backers DS, Erdmann AL. Formação do enfermeiro pelo olhar do empreendedorismo social. Rev Gaúcha Enferm [Internet]. 2009 [cited 2017 June 23];30(2):242-8. Available from: http://www.seer. ufrgs.br/index.php/RevistaGauchadeEnfermagem/ article/view/7252/6681

8. Ansari WE. Student nurse satisfaction levels witch their courses: Part I - effects of demographic variables. Nurse education Today [Internet]. 2002 [cited 2017 Sept 25];22(2):159-70. Available from: DOI: http://dx.doi.org/10.1054/nedt.2001.0682

9. Fontenele GM, Cunha RC. Formação pedagógica dos docentes de enfermagem em uma instituição superior privada na cidade de Parnaíba. Educ Linguagem [Internet]. 2014 [cited 2017 Sept 19];3(5):109-27. Available from: http://www.fecilcam.br/revista/index.php/ educacaoelinguagens/article/viewFile/670/405

10. Bos E, Alinaghizadeh H, Saarikoski M, Kaila P. Factors associated with student learning processes in primary health care units: A questionnaire study. Nurse Educ Today [Internet]. 2015 [cited 2017 Oct 13];35(1):170-5. Available from: Doi: http://linkinghub.elsevier.com/retrieve/pii/ s0260691714003335

11. Resende JC, Alves RBS, Coutinho MS, Bragagnoli G, Araujo CRF. Importância da iniciação científica e projetos de extensão para graduação em medicina. Rev bras ciênc saúde [Internet]. 2013 [cited 2017 Sept 18];17(1):11-8. Available from: http://periodicos.ufpb.br/index.php/rbcs/article/ view/14029/940

12. Oliveira CT, Santos AS. Percepções de estudantes universitários sobre a realização de atividades extracurriculares na graduação. Psicol ciênc prof [Internet]. 2016 [cited 2017 Sept 25];36(4):864-76. Available from: http://www.redalyc.org/pdf/2820/ 282048758007.pdf

13. Pereira AK, Koshino MF, Ferreira TR, Rocha RA. A importância das atividades extracurriculares universitárias para o alcance dos objetivos profissionais dos alunos de administração da Universidade Federal de Santa Catarina. Rev 
Gual [Internet]. 2011 [cited 2017 Sept 23];ed especial:163-94. Available from: DOI: http:// dx.doi.org/10.5007/1983-4535.2011v4nespp163

14. Cavalcanti CO, Costa MBS. Formação acadêmica em enfermagem: Implicações nas competências gerenciais do enfermeiro. Rev enferm UFPE [Internet]. 2013 [cited 2017 Sept 18];7(esp):7234-41. Available from: https://periodicos.ufpe.br/revistas/ revistaenfermagem/article/view/12400

15. Hernando CG, Martín MAC, Ortega FL, Villamor PM. Aprendizaje basado en problemas y satisfacción de los estudiantes de Enfermería. Enferm Glob [Internet]. 2014 [cited 2017 Sept 18];13(35):97-103. Available from: http:// scielo.isciii.es/scielo.php?script=sci_arttext\&pid= S1695-61412014000300006

16. Valbjorg E, Oddny I. Evaluation of students' satisfaction with nursing education in Norway. J Adv Nurs [internet]. 2003 [cited 2017Sept 19];42(3):226-36. DOI: 10.1046 / j.1365-2648.2003.02611.x

17. Puentes RV, Aquino OF, Quillici Neto A. Profissionalização dos professores: conhecimentos, saberes e competências necessárias à docência. Educar [internet]. 2009 [cited 2017 June 10];34:169-84. Available from: http://www.scielo. br/pdf/er/n34/10.pdf

18. Pereira FJR, Santos SR, Silva CC. Política de formação inclusiva: percepção de gestores sobre processo de mudanças em Instituições de Ensino Superior. Rev Bras Enferm [internet]. 2011 [cited 2017 June 10];64(4):711-6. Available from: http:// www.scielo.br/pdf/reben/v64n4/a13v64n4.pdf
19. Erdmann AL, Fernandes JD, Texeira GA. Panorama da educação em enfermagem no Brasil: graduação e pós-graduação. Enferm Foco [internet]. 2011 [cited 2017 June 10];2(supl):89-93. Available from: http://www.scielo.br/pdf/reben/v66nspe/ v66nspea14.pdf

20. Draganov PB, Sanna MC. Avaliação das competências de professores de enfermagem para desenvolver programas educativos para adultos. Rev Bras Enferm [internet]. 2013 [cited 2017 June 10];66(4):543-9. Available from: http://www.scielo. br/pdf/reben/v66n4/v66n4a12.pdf

21. Brasil. Ministério da Educação e Cultura. Conselho Nacional de Educação. Resolução CNE/ CES n. 3, de 7 de novembro de 2001. Institui Diretrizes curriculares do curso de graduação em enfermagem [Internet]. Brasília; 2001 [cited 2015 Dec 20]. Available from: http://portal.mec.gov.br/ cne/arquivos/pdf/CES03.pdf

22. Martins CB. A reforma universitária de 1968 e a abertura para o ensino superior privado no Brasil. Educ Soc [internet]. 2009 [cited 2017 Oct 15];30(106);15-35. Available from: http://www. scielo.br/pdf/es/v30n106/v30n106a02.pdf

23. Franco AP. Ensino superior no Brasil: cenário, avanços e contradições. J Políticas Educ [internet]. 2008 [cited 2017 Oct 16];4:53-63. Available from: http://revistas.ufpr.br/jpe/article/view/15028

Recebido: 30 de janeiro de 2018

Aprovado: 5 de março de 2018

Publicado: 25 de abril de 2018

A Revista Baiana de Enfermagem utiliza a Licença Creative Commons - Atribuição-NãoComercial 4.0 Internacional.

https://creativecommons.org/licenses/by-nc/4.0/

Este artigo é de acesso aberto distribuído sob os termos da Licença Creative Commons (CC BY-NC).

Esta licença permite que outros remixem, adaptem e criem a partir do seu trabalho para fins não comerciais e, embora, os novos trabalhos tenham de lhe atribuir o devido crédito e não possam ser usados para fins comerciais, os usuários não têm de licenciar esses trabalhos derivados sob os mesmos termos. 